PROCEEDINGS OF THE AMERICAN MATHEMATICAL SOCIETY

Volume 127, Number 3, March 1999, Pages 881-888

S 0002-9939(99)05192-8

\title{
CODIMENSION 2 NONFIBRATORS WITH FINITE FUNDAMENTAL GROUPS
}

\author{
R. J. DAVERMAN
}

(Communicated by Ralph Cohen)

\begin{abstract}
Fibrators are $n$-manifolds which automatically induce approximate fibrations, in the following sense: given any proper mapping $p$ from an $(n+k)$-manifold onto a finite-dimensional metric space such that, up to shape, each point-preimage is a copy of the fibrator, $p$ is necessarily an approximate fibration. This paper sets forth new examples, for the case $k=2$, of nonfibrators whose fundamental groups are finite.
\end{abstract}

Fibrators are manifolds which, in context, automatically induce approximate fibrations. The appropriate context involves a surjective, proper map $p: M \rightarrow B$ defined on a manifold $M$, under relatively mild restrictions on $B$, where all the point preimages have the shape (i.e., the approximate homotopy type) of a fixed closed, connected $n$-manifold $N$. The manifold $N$ is called a fibrator if all such maps $p$ are approximate fibrations. To appreciate the fibrator concept, it helps to regard approximate fibrations as beneficial; they are essentially as efficacious as the notable class of fibrations (see [CD1], [CD2] for evidence).

Nonfibrators are surprisingly scarce. The most obvious manifold to consider, the 1-sphere, fails to be a fibrator, due to a partition of the Möbius band into circles, and, perhaps even more familiarly, in codimension 2 due to the singularities typically present in Seifert fibrations. Somewhat similarly, the $n$-sphere, $n>1$, is a nonfibrator, but only in case the dimension of the supermanifold exceeds $2 n$. Among closed 2-manifolds merely the torus and Klein bottle fail to be fibrators in codimension 2 [D1]. No manifold $N$ admitting a regular self-covering map $N \rightarrow N$ with a cyclic group of covering transformations can be a codimension 2 fibrator [D1, Theorem 4.2]. Otherwise, known counterexamples in codimension 2 are relatively sporadic, arising from certain special bundles over $S^{1}$ whose fibers themselves are bundles over $S^{1}$; [D4, 6 ] presents concrete examples. Conversely, in the orientable setting, reasonable manifolds $N$ with nonzero Euler characteristic or hyperhopfian fundamental group are codimension 2 fibrators [D5].

This paper unveils new examples of codimension 2 nonfibrators with finite fundamental groups. It provides thereby a negative answer to a question raised in [D2] and treated, with only partial success, in [D3]. Unlike all previous constructions,

Received by the editors May 24, 1997.

1991 Mathematics Subject Classification. Primary 55R65, 57N15, 57N10; Secondary 57S37, $57 \mathrm{~N} 55$.

Key words and phrases. Approximate fibration, fibrator, homotopy equivalence, degree, local winding function, Lens space, hopfian manifold, locally flat.

This research was supported in part by NSF Grant DMS-9401086.

(c)1999 American Mathematical Society 
this necesarily entails PL knottedness. On the positive side, it establishes (Theorem 3.4) that manifolds having finite first homology and possessing a modest amount of homological asymmetry ordinarily are codimension 2 fibrators.

\section{Definitions}

An $n$-manifold is understood to be a separable metric space modelled on $\mathbb{R}^{n}$, and an $n$-manifold with boundary, a separable metric space modelled on the $n$-cell, $I^{n}$.

A proper map $p: M \rightarrow B$ between locally compact ANR's is called an approximate fibration if it has the following approximate homotopy lifting property: given an open cover $\Omega$ of $B$, an arbitrary space $X$, and two maps $f: X \rightarrow M$ and $F: X \times I \rightarrow B$ with $p f=F_{0}$, there exists a map $F^{\prime}: X \times I \rightarrow M$ such that $F_{0}^{\prime}=f$ and $p F^{\prime}$ is $\Omega$-close to $F$. The latter means: to each $z \in X \times I$ there corresponds $U_{z} \in \Omega$ such that $\left\{F(z), p F^{\prime}(z)\right\} \subset U_{z}$.

Fix a closed $n$-manifold $N$. A surjective, proper map $p: M \rightarrow B$ defined on a manifold $M$ is said to be $N$-like if each $p^{-1} b, b \in B$, has the same shape as $N$. Furthermore, $N$ is a codimension $k$ (orientable) fibrator if every $N$-like map $p: M \rightarrow B$ from a (respectively, orientable) $(n+k)$-manifold $M$ onto a finitedimensional metric space $B$ is an approximate fibration and $B$ necessarily is an ANR.

Given closed, connected $n$-manifolds $N$ and $N^{\prime}$, oriented by choices of generators $\xi \in H_{n}(N ; \mathbb{Z}), \xi^{\prime} \in H_{n}\left(N^{\prime} ; \mathbb{Z}\right)$, the degree of a map $f: N \rightarrow N^{\prime}$ is the integer $d$ such that $f_{*}(\xi)=d \cdot \xi^{\prime}$. A standard consequence of Poincaré duality promises that maps of degree \pm 1 induce epimorphisms at all homology levels.

Recall that a group $\Gamma$ is hopfian if every epimorphism $\Gamma \rightarrow \Gamma$ is an automorphism; more generally, $\Gamma$ is hyperhopfian if every homomorphism $\psi: \Gamma \rightarrow \Gamma$ with $\psi(\Gamma)$ normal and $\Gamma / \psi(\Gamma)$ cyclic is an automorphism.

A closed manifold $N$ is hopfian if it is orientable and if every degree one map $N \rightarrow N$ inducing a $\pi_{1}$-automorphism is a homotopy equivalence. The term aids in efficiently identifying approximate fibrations - cf. Theorem 3.2 here.

Consider an $N$-like map $p: M \rightarrow B$ defined on a manifold $M$, where $N$ is orientable. (The ensuing discussion presumes each point preimage to be an ANR; in the general case, where they merely have the shape of a manifold, the retractions and deformation retractions described below must be taken in a shape-theoretic sense, and the singular homology groups must be replaced with Čech homology groups.) There are useful winding functions defined locally on $B$ in the following way: each $b \in B$ has neighborhoods $U \supset U_{0}$ such that $p^{-1}(U)$ retracts to $p^{-1} b$ and $p^{-1}\left(U_{0}\right)$ strong deformation retracts to $p^{-1} b$ in $p^{-1}(U)$. Therefore, the inclusioninduced

$$
\psi_{b}: H_{n}\left(p^{-1} b ; \mathbb{Z}\right) \rightarrow H_{n}\left(p^{-1} U ; \mathbb{Z}\right)
$$

is an isomorphism onto the image of

$$
\psi: H_{n}\left(p^{-1} U_{0} ; \mathbb{Z}\right) \rightarrow H_{n}\left(p^{-1} U ; \mathbb{Z}\right) .
$$

For each $c \in U_{0}$ the image of

$$
\psi_{c}: H_{n}\left(p^{-1} c ; \mathbb{Z}\right) \rightarrow H_{n}\left(p^{-1} U ; \mathbb{Z}\right)
$$

is contained in $\operatorname{im}(\psi)$. Thus,

$$
\psi_{b}^{-1} \circ \psi_{c}: H_{n}\left(p^{-1} c ; \mathbb{Z}\right) \rightarrow H_{n}\left(p^{-1} b ; \mathbb{Z}\right)
$$


is a well-defined homomorphism between two copies of $\mathbb{Z}$, meaning that (up to sign) it amounts to multiplication by some integer $q_{c} \geq 0$. The local winding function $\alpha_{b}: U_{0} \rightarrow \mathbb{Z}$ at $b$ is defined by the rule $\alpha_{b}(c)=q_{c}$. The continuity set $C$ of $p$ consists of those $b \in B$ such that $\alpha_{b}$ is continuous in some neighborhood of $b$; in other words, the retractions $p^{-1} U \rightarrow p^{-1} b$ mentioned above restrict to maps $p^{-1} c \rightarrow p^{-1} b$ of degree \pm 1 for all $c \in B$ sufficiently close to $b$. According to [CD3, Proposition 3], $C$ is an open, dense subset of $B$.

When studying $N$-like maps $p: M \rightarrow B, N$ nonorientable, one can use $\mathbb{Z}_{2}$ coefficients to define completely analogous notions of winding functions and continuity set. The latter will be called the mod 2 continuity set, for emphasis.

\section{EXAMPLES}

Example 2.1. A 6-manifold with finite $\pi_{1}$ which is NOT a codimension 2 orientable PL fibrator.

Let $\Gamma_{m}$ denote the cyclic group of odd order $m>2$. The manifold will be $L_{m, q} \times S^{3}$, where $L_{m, q}$ is any 3-dimensional Lens space having fundamental group $\Gamma_{m}$.

Build a 5-manifold with boundary $M^{5}$ partitioned into copies of $L_{m, q}$ and a single (knotted) 3 -sphere $S$, equipped with a semi-free $\Gamma_{m}$ action which is pointwise fixed on $S$ and which permutes those copies of the Lens spaces comprising the partition. To get started, find a knotted 2-sphere $K$ in $S^{4}$ whose complement is fibered by punctured Lens spaces - namely, objects equivalent to the closure of $\left(L_{m, q} \backslash\right.$ PL 3-cell). For any choice of $m$ (odd) and $q$, Zeeman [Z] shows how to get a knotted PL 2-sphere $K$ in $S^{4}$ whose (compactified) complement is fibered by the punctured $L_{m, q}$; Giffen [G] has a slightly different perspective on the construction which clarifies the existence of a semifree $\Gamma_{m}$ action fixing $K$. Just as in [DW, §5], $M^{5}$ arises by attaching a 3 -handle to $B^{5}$ along a regular neighborhood of $K$ in $\partial B^{5}$. The attaching is regulated throughout this regular neighborhood to superimpose parallel copies of the attaching sphere atop boundaries of the punctured Lens spaces. The 3 -sphere, $S$, equals the core of the 3 -handle plus the cone (in $B^{5}$ ) over $K$. The Lens spaces in $\partial M^{5}$ arise by adjoining 3-balls parallel to the handle core to the various punctured Lens spaces; those in the interior run parallel to those in $\partial M^{5}$. The $\Gamma_{m}$ action on $M^{5}$ is determined on $B^{5}$ by coning over the given action on $S^{4}=\partial B^{5}$, and then on the 3 -handle it is determined to fix the core and to extend the action of the attaching region so as to permute the (unpunctured) Lens spaces.

Endow $S^{3}$ with the standard free $\Gamma_{m}$ action having $L_{m, q}$ as orbit space. Form a new manifold $\left(\operatorname{Int} M^{5}\right) \times S^{3}$ with (free) diagonal $\Gamma_{m}$ action, and let $M^{8}$ denote its orbit space. It follows that $M^{8}$ is partitioned into copies of $L_{m, q} \times S^{3}-$ the images of the various $L \times S^{3}$ ( $L$ a Lens space from the partition), which descend homeomorphically - and the image of $S \times S^{3}$, which in turn is homeomorphic to $S^{3} \times L_{m, q}$, since the diagonal action fixes all first coordinate points. One can easily confirm that the quotient map for this partition fails to be an approximate fibration, precisely because its continuity set excludes the image of $S \times S^{3}$.

Theorem 2.2. Suppose $L^{n}$ is an $n$-dimensional Lens space such that $\pi_{1}\left(L^{n}\right) \cong$ $\Gamma_{m}$, where $m$ is odd and $n$ is congruent to 3 modulo 4 . Then $L^{n} \times S^{n}$ fails to be a codimension 2 orientable fibrator.

Proof. The heart of the construction, like the preceding for $n=3$, involves production of a knotted $(n-1)$-sphere $K$ (locally flatly) embedded in $S^{n+1}=\partial B^{n+2}$ 
whose complement is fibered by punctured copies of $L^{n}$ and which itself is the fixed point set of a semifree $\Gamma_{m}$ action on $S^{n+1}$. Once all this structure is arranged, the rest of the argument coincides with that of Example 2.1 above.

Write $n=4 k-1$. Regard $S^{n}$ as the join of $2 k$ copies of $S^{1}$, and $L_{n}$ as the quotient of a $\Gamma_{m}$ action operating via rotation of period $m$ on each circle factor in this join. Name an involution $\phi: S^{n} \rightarrow S^{n}$ determined by reflection in each circle factor; $\phi$ covers a degree one involution $\Phi: L^{n} \rightarrow L^{n}$ leaving some $n$-cell $D$ invariant and acting by inversion on $\pi_{1}(L)$. Let $L^{*}=L^{n} \backslash$ IntD, and form the mapping torus $\operatorname{Map}\left(\Phi^{*}\right)$ of $\Phi \mid L^{*}$, namely, the space resulting from $L^{*} \times[0,1]$ under identification of each $\langle x, 1\rangle$ with $\left\langle\Phi^{*}(x), 0\right\rangle$. $\operatorname{Map}\left(\Phi^{*}\right)$ admits a natural free $\Gamma_{m}$ action, generated by the map sending the class of $\langle x, t\rangle$ to that of $\left\langle x, t^{\prime}\right\rangle$, where $t^{\prime} \in[0,1]$ is congruent to $t+(2 / m)$ modulo 1 ; as $\Phi^{2}=$ Identity, this yields a $\Gamma_{m}$ action (indeed, the same idea affords a free $\Gamma_{2 d+1}$ action on $\operatorname{Map}\left(\Phi^{*}\right)$ for all $d \geq 0$, but only $\Gamma_{m}$ is significant here). Since $\Phi^{*} \mid \partial L^{*}$ has degree 1, it is isotopic to the identity, which implies $\partial\left(\operatorname{Map}\left(\Phi^{*}\right)\right) \approx S^{1} \times S^{n-1}$. We concoct a new (PL or smooth) manifold $Y$ by attaching $B^{2} \times S^{n-1}$ to $\operatorname{Map}\left(\Phi^{*}\right)$ via an obvious homeomorphism between boundaries. As Giffen $[G]$ described, the free action on $\operatorname{Map}\left(\Phi^{*}\right)$ extends to a semifree $\Gamma_{m}$ action on $Y$ fixing the $(n-1)$-sphere $K=0 \times S^{n-1} \subset B^{2} \times S^{n-1}$.

The claim, of course, is that $Y \approx S^{n+1}$. Routine computation indicates $\pi_{1}(Y)$ is trivial (here $\pi_{1}\left(\operatorname{Map}\left(\Phi^{*}\right)\right)$ has presentation

$$
\left\langle\zeta, s: s \zeta s^{-1}=\zeta^{-1}, \zeta^{m}=1\right\rangle ;
$$

upon inclusion in $Y=\operatorname{Map}\left(\Phi^{*}\right) \cup B^{2} \times S^{n-1}, s$ is nullhomotopic, leading to the presentation

$$
\left.\pi_{1}(Y)=\left\langle\zeta: \zeta^{2}=1=\zeta^{m}\right\rangle \cong 1\right) .
$$

Furthermore, $Y$ has the homology of $S^{n+1}$. One confirmation exploits the Wang exact sequence of the fibration $L^{*} \hookrightarrow \operatorname{Map}\left(\Phi^{*}\right) \rightarrow S^{1}$ to compute the cohomology of $\operatorname{Map}\left(\Phi^{*}\right)$ with $\Gamma_{m}$ coefficients:

$$
\cdots \rightarrow H^{q}\left(\operatorname{Map}\left(\Phi^{*}\right)\right) \rightarrow H^{q}\left(L^{*}\right) \stackrel{\theta}{\rightarrow} H^{q}\left(L^{*}\right) \rightarrow H^{q+1}\left(\operatorname{Map}\left(\Phi^{*}\right)\right) \rightarrow \cdots
$$

where $\theta(u \smile v)=\theta(u) \smile v+u \smile \theta(v)$. The analysis of $\pi_{1}\left(\operatorname{Map}\left(\Phi^{*}\right)\right)$ implies $H_{1}\left(\operatorname{Map}\left(\Phi^{*}\right) ; \mathbb{Z}\right) \cong \mathbb{Z} ;$ by Universal Coefficient Theorems, $\left.H_{1}\left(\operatorname{Map}\left(\Phi^{*}\right) ; \Gamma_{m}\right)\right) \cong$ $\Gamma_{m}$. Since each $H^{q}\left(L^{*}\right)$ is in the subring generated by $H^{1}\left(L^{*}\right)$, one can combine the formula for $\theta$ with the conclusion that $\theta$ acts isomorphically on $H^{1}\left(L^{*}\right)$ (deduced from the data that each cohomology group in this Wang sequence at levels 0 and 1 is a copy of $\Gamma_{m}$ ) to discover that $\theta$ acts isomorphically on all cohomology above level 0 . Hence, $H^{q}\left(\operatorname{Map}\left(\Phi^{*}\right) ; \Gamma_{m}\right) \cong 0$ whenever $q>1$. Express $\operatorname{Map}\left(\Phi^{*}\right)$ as a union of two copies of $L^{*} \times[0,1]$; the associated Mayer-Vietoris sequence gives that elements of $H^{q}\left(\operatorname{Map}\left(\Phi^{*} ; \mathbb{Z}\right)\right), q>1$, have order $m$. Universal Coefficient computations then give that $\operatorname{Map}\left(\Phi^{*}\right)$ has the integral homology of $S^{1}$, from which the conclusion about the homology of $Y$ follows. That $Y$ then is (smoothly or PL) equivalent to $S^{n+1}$ stems from the high dimensional Poincaré Conjecture [RS, p. 9].

The methods employed here do not address the following unsolved question, first raised in [D4]: Is each Lens space a codimension 2 fibrator? If so, the property of being a codimension 2 fibrator would not be preserved under the operation of Cartesian product. In the next section we will see that some Lens spaces are codimension 2 fibrators, but their dimensions are congruent to 1 modulo 4 , so they have no overlap with the ones mentioned in Theorem 2.2 . 


\section{Conditions YiElding APPROXimate FiBRAtions}

For convenience and efficiency, we begin by restating two key results about structures surrounding $N$-like mappings.

Theorem 3.1 ([DW, Theorem 4.1], [D1, Theorem 3.1 \& Proposition 4.1]). Suppose $N$ is a closed, connected n-manifold and $p: M^{n+2} \rightarrow B$ an $N$-like map. When $M$ and $N$ are both orientable, $B$ is a 2-manifold and $D=B \backslash C$ is locally finite in $B$ (where $C$ denotes the continuity set of $p$ ); otherwise, $B$ is a 2-manifold with boundary, and $D^{\prime}=\operatorname{Int} B \backslash C^{\prime}$ is locally finite in Int $B$ (where $C^{\prime}$ denotes the mod 2 continuity set of $p$ ).

Theorem 3.2 ([D2, Proposition 2.7]). Let $N$ be a closed, connected, orientable, hopfian n-manifold with hopfian fundamental group, and let $p: M^{n+2} \rightarrow B$ be an $N$-like map defined on an $(n+2)$-manifold $M$. Then $p$ restricts to an approximate fibration on $p^{-1}(C)$.

Remark. The result claimed here, which is a bit more general than the one cited, follows by the same argument.

Lemma 3.3. Let $N$ be a closed, connected, orientable $n$-manifold with finite first homology group, and let $p: M \rightarrow \mathbb{R}^{2}$ be an $N$-like map defined on an $(n+2)$ manifold $M$ that restricts to an approximate fibration on $M \backslash p^{-1}$ (origin). Then the continuity set of $p$ equals $\mathbb{R}^{2}$ if and only if $H_{1}(M) \cong \mathbb{Z} \oplus H_{1}(N)$.

Proof. Throughout the remainder of this section we use $O$ to denote the origin in $\mathbb{R}^{2}$. Since $p$ is an approximate fibration over $\mathbb{R}^{2} \backslash\{O\}$, the homotopy exact sequence for the approximate fibration [CD1, Corollary 3.5] shows that $\pi_{1}\left(M \backslash p^{-1}(O)\right)$ is an extension of $\pi_{1}(N)$ by $\mathbb{Z}$. In particular, $H_{1}\left(M \backslash p^{-1}(O)\right)$ is infinite. Also, $p^{-1}(O)$ is a (shape) deformation retract of $M$. (To check this claim, given a neighborhood $U$ of $p^{-1}(O)$, construct a homotopy $h_{t}$ of $\mathbb{R}^{2}$ starting at the identity, ending with a map into $\mathbb{R}^{2} \backslash p(M \backslash U)$, fixing a neighborhood of $O$, and preserving $\mathbb{R}^{2} \backslash\{O\}$ throughout. Since $p$ is an approximate fibration over $\mathbb{R}^{2} \backslash\{O\}$, by [CD1, Proposition 1.5] $h_{t}$ can be approximately lifted to a homotopy of $M$ starting at the identity, ending with a map into $U$, fixing a neighborhood of $p^{-1}(O)$, and preserving $M \backslash p^{-1}(O)$ throughout.) Hence, $H_{1}(M) \cong H_{1}\left(p^{-1}(O)\right) \cong H_{1}(N)$. Examine the following part (*) of the long exact homology sequence for the pair $\left\{M, M \backslash p^{-1}(O)\right\}$ :

$(*) \quad H_{2}\left(M, M \backslash p^{-1}(O)\right) \rightarrow H_{1}\left(M \backslash p^{-1}(O)\right) \rightarrow H_{1}(M) \rightarrow H_{1}\left(M, M \backslash p^{-1}(O)\right)$.

Here, by duality, $H_{2}\left(M, M \backslash p^{-1}(O)\right) \cong H^{n}\left(p^{-1}(O)\right) \cong \mathbb{Z}$ and $H_{1}\left(M, M \backslash p^{-1}(O)\right) \cong$ $H^{n+1}\left(p^{-1}(O)\right) \cong 0$, while the deformation retraction $R: M \rightarrow p^{-1}(O)$ can be regarded as inducing an isomorphism $H_{1}(M) \rightarrow H_{1}(N)$. Thus, (*) reduces to

$$
\mathbb{Z} \rightarrow H_{1}\left(M \backslash p^{-1}(O)\right) \rightarrow H_{1}(N) \rightarrow 0 .
$$

Assume that $C$, the continuity set of $p$, equals $\mathbb{R}^{2}$. For all $z \in \mathbb{R}^{2} \backslash\{O\}$, $H_{1}\left(p^{-1} z\right) \rightarrow H_{1}(M) \cong H_{1}(N)$ is an isomorphism factoring through $H_{1}\left(M \backslash p^{-1}(O)\right)$, implying that $H_{1}\left(M \backslash p^{-1}(O)\right)$ has $H_{1}(N)$ as a direct summand. In view of the assurance that $H_{1}\left(M \backslash p^{-1}(O)\right)$ is infinite, diagram chasing in (**) above yields $H_{1}\left(M \backslash p^{-1}(O)\right) \cong \mathbb{Z} \oplus H_{1}(N)$.

Conversely, assume $H_{1}\left(M \backslash p^{-1}(O)\right) \cong \mathbb{Z} \oplus H_{1}(N)$. In this case (**) becomes

$$
\mathbb{Z} \rightarrow \mathbb{Z} \oplus H_{1}(N) \rightarrow H_{1}(N) \rightarrow 0
$$


where the $H_{1}(N)$ summand in the second term is carried by any fiber $p^{-1}(z) \subset$ $M \backslash p^{-1}(O)$, because $\pi_{1}\left(M \backslash p^{-1}(O)\right)$ is known to be an extension of $\pi_{1}(N)$ by $\mathbb{Z}$. As $H_{1}(N)$ is torsion, inspection of $(*)$ confirms that $\partial: H_{2}\left(M, M \backslash p^{-1}(O)\right) \rightarrow H_{1}(M \backslash$ $\left.p^{-1}(O)\right)$ is 1-1 and, therefore, the inclusion-induced $H_{1}\left(M \backslash p^{-1}(O)\right) \rightarrow H_{1}(M)$ restricts to an isomorphism $H_{1}\left(p^{-1} z\right) \rightarrow H_{1}(M) \cong H_{1}\left(p^{-1}(O)\right)$. By [Im, Lemma 3.2] (or see [D5, Lemma 5.2]) $C=\mathbb{R}^{2}$.

We define a special class Asym of homologically asymmetric (closed, connected) manifolds: $N \in$ Asym if it is orientable and each homotopy equivalence $N \rightarrow N$ of degree +1 induces the identity automorphism $H_{1}(N) \rightarrow H_{1}(N)$.

Theorem 3.4. Let $N^{n} \in$ Asym be a hopfian manifold such that $H_{1}\left(N^{n}\right)$ is finite and $\pi_{1}\left(N^{n}\right)$ is a hopfian group. Then $N^{n}$ is a codimension 2 orientable fibrator.

Simple examples like $S^{1} \in$ Asym clarify the need for the finite $H_{1}$ hypothesis in 3.4 .

Corollary 3.5. Every $N^{n} \in$ Asym for which $\pi_{1}(N)$ is finite is a codimension 2 orientable fibrator.

Corollary 3.6. Some Lens spaces are codimension 2 fibrators.

Proof. Among Lens spaces of dimension $n=4 k+1$, some belong to Asym - they are characterized by having all units $a \in \Gamma_{m} \cong \pi_{1}(L)$ satisfy $a^{2 k+1} \equiv+1 \bmod m$ [Co, 29.6]. (When $n=4 k-1$, however, as in 2.2 there always exists a degree 1 map $F: L \rightarrow L$ such that $F_{*}(\zeta)=\zeta^{-1}$ for all $\zeta \in H_{1}(L)$.) Restrict attention to those Lens spaces in Asym with odd order fundamental groups. Then any such example in a nonorientable $(n+2)$-manifold has an orientable neighborhood [Ch1, Proposition 3.2 ], which quickly promotes the conclusion of 3.5 about being a codimension 2 orientable fibrator to the nonorientable case.

In a related vein, Chinen [Ch2] has shown every Lens space with fundamental group of order equal to a power of 2 is a codimension 2 fibrator.

Proof of 3.4. In light of 3.2, it suffices to show each $N^{n}$-like map $p: M \rightarrow B$ has $C$ $=B$. Using 3.1, we localize to the setting where $B=\mathbb{R}^{2}$ and $p$ is an approximate fibration over $\mathbb{R}^{2} \backslash\{O\}$. The point will be that $M \backslash p^{-1}(O)$ has the homotopy type of the mapping torus of a degree +1 homotopy equivalence $g: N^{n} \rightarrow N^{n}$; a straightforward computation, based on the hypothesis $N^{n} \in A$ sym, then will give $H_{1}\left(M \backslash p^{-1}(O)\right) \cong \mathbb{Z} \oplus H_{1}\left(N^{n}\right)$, and 3.3 will complete the argument.

Let $S^{1}$ denote the unit circle in $\mathbb{R}^{2}$ centered at $O$. Approximate homotopy lifting features imply $p^{-1}\left(S^{1}\right)$ has the same shape as the mapping torus of some homotopy equivalence $g: N^{n} \rightarrow N^{n}$. If $g$ had degree -1 instead of the desired degree, a straightforward cohomology computation would yield $\check{H}^{n+1}\left(p^{-1}\left(S^{1}\right)\right) \cong$ $\mathbb{Z}_{2}$. Using duality in $T=M \backslash p^{-1}(O)$ and part of the homology exact sequence for $\left\{T, T \backslash p^{-1}\left(S^{1}\right)\right\}$,

$$
\check{H}^{n+1}\left(p^{-1}\left(S^{1}\right)\right) \cong H_{1}\left(T, T \backslash p^{-1}\left(S^{1}\right)\right) \rightarrow \widetilde{H}_{0}\left(T \backslash p^{-1}\left(S^{1}\right)\right) \cong \mathbb{Z} \rightarrow 0 \cong \widetilde{H}_{0}(T),
$$

we would reach the contradiction that $\check{H}^{n+1}\left(p^{-1}\left(S^{1}\right)\right)$ is infinite.

The examples of $\S 2$ tolerate PL wildness; wildness crops up upon coning over a knotted codimension 2 sphere. We close by establishing the necessity of wildness in such counterexamples. 
Lemma 3.7. If $p: M \rightarrow \mathbb{R}^{2}$ is an approximate fibration over $\mathbb{R}^{2} \backslash\{O\}$ and $Q=$ $p^{-1}(O)$ is a compact, locally flatly embedded, simply connected $n$-manifold, then $\pi_{1}(M \backslash Q)$ is cyclic.

Proof. By [KS], $Q$ has a neighborhood $U$ in $M$ which is a locally trivial open disk bundle over $Q$. The homotopy exact sequence of the induced punctured disk bundle with total space $U \backslash Q$ and base $Q$,

$$
\left.\mathbb{Z} \cong \pi_{1} \text { ( punctured disk bundle }\right) \rightarrow \pi_{1}(U \backslash Q) \rightarrow \pi_{1}(Q) \cong 0,
$$

reveals that $\pi_{1}(U \backslash Q)$ is cyclic. Repeating the technique outlined in a parenthetical note early in the proof of 3.3, we can apply the regular approximate homotopy lifting property for $p \mid M \backslash Q$ [CD1, Proposition 1.5] to produce a homotopy of $M$ starting at the identity, ending with a map into $U$, fixing a neighborhood of $Q$, and preserving $M-Q$ throughout. This means $\pi_{1}(M \backslash Q)$ is a retract of $\pi_{1}(U \backslash Q)$, and consequently it must be cyclic as well.

Theorem 3.8. Let $N$ be a closed, orientable $n$-manifold with finite fundamental group, $B$ a 2-manifold, and $p: M^{n+2} \rightarrow B$ a surjective mapping defined on an $(n+2)$-manifold $M$ such that each $p^{-1} b$ is an $n$-manifold homotopy equivalent to $N$ and locally flatly embedded in $M$. Then $p$ is an approximate fibration.

Proof. Without loss of generality restrict to the setting where $B=\mathbb{R}^{2}$ and $p$ is an approximate fibration over $\mathbb{R}^{2} \backslash\{O\}$. Then there exists a retraction $R: M \rightarrow p^{-1}(O)$ (n.b., a genuine retraction, not just a shape retraction, since $p^{-1}(O)$ really is a manifold). Fix $z \in \mathbb{R}^{2} \backslash\{O\}$.

Let $\Theta: M^{\prime} \rightarrow M$ denote the universal covering. Here $M^{\prime}$ is partitioned into codimension 2 manifolds via the components of $(p \Theta)^{-1}(b), b \in B$. As $(p \Theta)$ is an approximate fibration over $\mathbb{R}^{2} \backslash\{O\}$ [D2, Lemma 2.5], $(p \Theta)^{-1}(O)$ is a strong deformation retract of $M^{\prime}$. By construction $Q=(p \Theta)^{-1}(O)$ is simply connected; it is also locally flatly embedded, since its image under $\Theta$ is.

Suppose $p$ fails to be an approximate fibration. Then $R \mid p^{-1} z: p^{-1} z \rightarrow p^{-1}(O)$ cannot induce a surjection at the fundamental group level, for otherwise again the continuity set of $p$ would equal $\mathbb{R}^{2}[\mathrm{Im}$, Lemma 3.2] and Theorem 3.2 here would imply $p$ is an approximate fibration. Let $N^{\prime}$ denote the cover of $N$ corresponding to $\operatorname{ker}\left(R \mid p^{-1} z\right)_{\#}$, under the equivalence $p^{-1} z \leftrightarrow N$; the nontriviality of $\pi_{1}\left(N^{\prime}\right)$ is a crucial feature. Moreover, $M^{\prime}$ is partitioned into copies of $N^{\prime}$ and the simplyconnected manifold $Q$. Let $q: M^{\prime} \backslash Q \rightarrow B^{\prime}$ denote the quotient map determined by the restricted partition. Applying [D2, Lemma 2.5] locally, we find that $q$ is an approximate fibration, and then we can readily check that $B^{\prime}$ is a covering space of $\mathbb{R}^{2} \backslash\{O\}$. Finally, from the homotopy exact sequence of $q \mid M^{\prime} \backslash Q[\mathrm{CD} 1$, Corollary 3.5] we deduce that $\pi_{1}\left(M^{\prime} \backslash Q\right)$ is an extension of $\pi_{1}\left(N^{\prime}\right)$ by $\mathbb{Z} \cong \pi_{1}\left(B^{\prime}\right)$. As this group cannot be cyclic, we have a contradiction to Lemma 3.7 .

\section{REFERENCES}

[Ch1] N. Chinen, Manifolds with nonzero Euler characteristic and codimension 2 fibrators, Topology Appl. 86 (1998), 151-167. CMP 98:12

[Ch2] ㄴ. Finite groups and approximate fibrations, preprint.

[Co] M. M. Cohen, A Course in Simple-Homotopy Theory, Springer-Verlag, New York, 1973. MR 50:14762

[CD1] D. S. Coram and P. F. Duvall, Approximate fibrations, Rocky Mountain J. Math. 7 (1977), 275-288. MR 56:1296 
[CD2] _ Approximate fibrations and a movability condition for maps, Pacific J. Math. 72 (1977), 41-56. MR 57:7597

[CD3] _ Nondegenerate $k$-sphere mappings between spheres, Topology Proceedings 4 (1979), 67-82. MR 81 m:57008

[D1] R. J. Daverman, Decompositions into codimension 2 manifolds: The nonorientable case, Topology Appl. 24 (1986), 71-81. MR 88a:57028

[D2] _ Submanifold decompositions that induce approximate fibrations, Topology Appl. 33 (1989), 173-184. MR 91d:57013

[D3] _ Manifolds with finite first homology as codimension 2 fibrators, Proc. Amer. Math. Soc. 113 (1991), 471-477. MR 92a:55015

[D4] _ 3-Manifolds with geometric structure and approximate fibrations, Indiana Univ. Math. J. 40 (1991), 1451-1469. MR 92m:55016

[D5] _ Hyperhopfian groups and approximate fibrations, Compositio Math. 86 (1993), 159-176. MR 94b:55022

[DW] R. J. Daverman and J. J. Walsh, Decompositions into codimension 2 manifolds, Trans. Amer. Math. Soc. 288 (1985), 273-291. MR 87h:57019

[G] C. H. Giffen, The generalized Smith conjecture, Amer. J. Math. 88 (1966), 187-198. MR 33:6620

[Im] Y. H. Im, Products of surfaces that induce approximate fibrations, Houston J. Math. 21 (1995), 339-348. MR 96c:57038

[KS] R. C. Kirby and L. C. Siebenmann, Normal bundles for codimension 2 locally flat embeddings, in Geometric Topology (L. C. Glaser and T. B. Rushing, eds.), Lecture Notes in Mathematics, vol. 438, Springer-Verlag, Berlin, 1975, pp. 310-324. MR 53:4072

[RS] C. Rourke and B. J. Sanderson, Introduction to Piece-wise Linear Topology, SpringerVerlag, Berlin, 1972. MR 50:3236

[Z] E. C. Zeeman, Twisting spun knots, Trans. Amer. Math. Soc. 115 (1965), 471-495. MR 33:3290

Department of Mathematics, University of Tennessee, Knoxville, Tennessee 37996 1300

E-mail address: daverman@novell.math.utk.edu 